\title{
Alternative Splicing as a Regulator of Early Plant Development
}

\author{
Dóra Szakonyi* and Paula Duque* \\ Instituto Gulbenkian de Ciência, Oeiras, Portugal
}

Most plant genes are interrupted by introns and the corresponding transcripts need to undergo pre-mRNA splicing to remove these intervening sequences. Alternative splicing (AS) is an important posttranscriptional process that creates multiple mRNA variants from a single pre-mRNA molecule, thereby enhancing the coding and regulatory potential of genomes. In plants, this mechanism has been implicated in the response to environmental cues, including abiotic and biotic stresses, in the regulation of key developmental processes such as flowering, and in circadian timekeeping. The early plant development steps - from embryo formation and seed germination to skoto- and photomorphogenesis - are critical to both execute the correct body plan and initiate a new reproductive cycle. We review here the available evidence for the involvement of AS

OPEN ACCESS

Edited by:

Simon Gilroy,

University of Wisconsin-Madison,

United States

Reviewed by:

Ligeng Ma,

Capital Normal University, China

Lydia Gramzow,

Friedrich-Schiller-Universität Jena,

Germany

*Correspondence:

Dóra Szakonyi

dszakonyi@igc.gulbenkian.pt;

Dora.Szakonyi@gmail.com

Paula Duque

duquep@igc.gulbenkian.pt

Specialty section:

This article was submitted to

Plant Cell Biology,

a section of the journal

Frontiers in Plant Science

Received: 03 April 2018

Accepted: 23 July 2018

Published: 15 August 2018

Citation:

Szakonyi D and Duque P (2018) Alternative Splicing as a Regulator

of Early Plant Development.

Front. Plant Sci. 9:1174.

doi: 10.3389/fpls.2018.01174 and various splicing factors in the initial stages of plant development, while highlighting recent findings as well as potential future challenges.

Keywords: alternative splicing, early seedling development, embryogenesis, photomorphogenesis, seed dormancy, seed maturation, seed germination, splicing factors

\section{mRNA PROCESSING AND ALTERNATIVE SPLICING}

Accurate processing of precursor mRNAs (pre-mRNAs) is a major step in gene expression crucial for performing everyday housekeeping functions, executing developmental programs, and responding to intrinsic and environmental cues. It involves modification steps to remove noncoding sequences as well as add the cap and the poly(A) tail to the $5^{\prime}$ and $3^{\prime}$ ends of the mRNA, respectively (reviewed in Proudfoot, 2011; Shi and Manley, 2015; Ramanathan et al., 2016). Pre-mRNA splicing, the excision of introns followed by joining of exons, is catalyzed by the spliceosome, a large ribonucleoprotein complex. The spliceosomal subunits assemble at conserved nucleotides at the exon-intron boundaries also known as the $5^{\prime}$ (or donor) and $3^{\prime}$ (or acceptor) splice sites (SS), the branch point and the polypyrimidine tract. In addition to the core spliceosomal components, many RNA-binding proteins play key roles in mRNA processing, SS selection and splicing (reviewed in Meyer et al., 2015). In higher eukaryotes, intron-containing genes frequently give rise to multiple mRNAs through alternative splicing (AS) (Figure 1A), during which differential recognition of SS can lead to intron retention, exon skipping and/or alternative $5^{\prime} / 3^{\prime}$ SS selection. AS can significantly enhance a genome's coding capacity by producing protein variants with altered function. It also often affects mRNA stability by introducing premature stop codons in the coding sequence, thus targeting these transcripts to degradation by nonsensemediated decay (NMD). Furthermore, AS can modify gene expression by modulating transcription elongation and/or translation efficiency (reviewed in Reddy et al., 2013; Laloum et al., 2017). It is hence not surprising that AS fulfills important biological functions. In plants, it has been found to control key processes like the circadian clock or flowering time as well as the response to environmental cues, including abiotic stress or pathogen attack (reviewed in Staiger and Brown, 2013; Yang et al., 2014; Laloum et al., 2017; Shang et al., 2017). 


\section{EARLY PLANT DEVELOPMENT}

The first stages of a plant's life are essential to establish the basic body pattern, develop different tissue types and initiate a new reproductive cycle (Figure 1B). Sexual reproduction of land plants involves the alternation of haploid and diploid stages. Angiosperms have a dominant diploid sporophyte and a relatively short haploid phase consisting of a few microscopic cells. Seeds are produced by double fertilization. One sperm cell fuses with the egg cell to form the diploid embryo, while a second sperm cell fertilizes the diploid central cell to give rise to the endosperm (reviewed in Raghavan, 2003; Berger et al., 2008). During embryogenesis, the one-cell zygote undergoes a tightly regulated developmental program to form a mature embryo. In dicots such as Arabidopsis thaliana (arabidopsis), this process includes distinct morphological stages, called globular, heart, torpedo, and bent cotyledon, leading to the establishment of the basic body plan and main tissue/organ initials including the shoot and root apical meristems (reviewed in Palovaara et al., 2016). Embryo morphogenesis is followed by seed maturation, which involves the accumulation of reserves, acquisition of desiccation tolerance, reduction of metabolic activities and induction of dormancy to enable survival of the embryo until favorable environmental conditions allow germination (reviewed in Graeber et al., 2012). Fresh seeds usually show high dormancy that gradually decreases over time in a process called after-ripening. The release from dormancy depends on environmental factors (e.g., light quality, day length, temperature, water availability, exposure to cold) and internal regulators (e.g., hormones, regulatory proteins, chromatin status) (reviewed in Kucera et al., 2005; Nee et al., 2017). Germination starts with water uptake (imbibition) and rapid expansion of the embryo, leading to rupture of the seed coat and emergence of the radicle. Seedlings growing in the dark display skotomorphogenic development (etiolated growth), characterized by elongated hypocotyls, apical hook, pale cotyledons and short roots. When exposed to light, the seedling undergoes photomorphogenesis to activate vegetative growth, displaying shorter and thicker hypocotyls as well as green and expanded cotyledons (reviewed in $\mathrm{Wu}, 2014)$. Hormones are important regulators of early plant development. Embryo formation is governed by auxins and cytokinins, while abscisic acid (ABA) is important for the completion of seed maturation and building up dormancy. $\mathrm{ABA}$ is also the major inhibitor of seed germination, with its effect being counteracted by gibberellic acid, ethylene, and brassinosteroids (reviewed in Palovaara et al., 2016).

\section{GLOBAL ALTERNATIVE SPLICING CHANGES DURING EARLY PLANT DEVELOPMENT}

Next-generation sequencing has revolutionized transcriptomic studies. The latest RNA-seq data gathered in higher plants showed that traditional approaches largely underestimated the proportion of genes undergoing AS. Current assessments indicate that up to $70 \%$ of plant multiexon genes generate more than one transcript via this mechanism, with intron retention representing the predominant mode of AS (Lu et al., 2010; Zhang et al., 2010; Marquez et al., 2012; Shen et al., 2014; Thatcher et al., 2014; Chamala et al., 2015; Sun and Xiao, 2015; Iniguez et al., 2017; Zhang et al., 2017). In fact, increased sequencing coverage revealed a large number of non-annotated AS events and splice variants (Marquez et al., 2012; Zhang et al., 2017). Most of the plant AS events map to coding regions, thereby altering protein sequence and potentially function or compromising mRNA stability. Indeed, a significant proportion of intron-containing genes are potentially regulated by NMD (Zhang et al., 2010; Kalyna et al., 2012; Drechsel et al., 2013). Although thousands of alternatively spliced mRNAs are detected in genome-wide analyses, detailed genetic and molecular studies will be required to identify functionally relevant AS events.

Numerous plant large-scale studies have focused on gene expression and AS patterns in different tissues and during development, identifying many novel organ- or stage-specific mRNAs with dynamic expression changes and a stage-dependent switch in isoform dominance for many genes (Zhang et al., 2010; Thatcher et al., 2014; Klepikova et al., 2016; Vaneechoutte et al., 2017). Notably, genes encoding alternatively spliced transcripts are not necessarily differentially expressed during developmental transitions, suggesting that AS shapes the transcriptome independently from transcriptional regulation (Srinivasan et al., 2016). These findings are confirmed by deepsequencing studies tracking expression and AS changes during the first stages of plant development (Aghamirzaie et al., 2013; Lu et al., 2013; Sun and Xiao, 2015; Qu et al., 2016; Thatcher et al., 2016; Narsai et al., 2017). The detection of prominent AS switches and of development-specific splice variants corroborates an important regulatory layer of early plant development at the splicing level. Interestingly, RNA-processing factors themselves undergo AS resulting in a potential autoregulatory feedback loop.

During embryogenesis in soybean (Aghamirzaie et al., 2013), AS of 47,331 genes produced 217,371 different transcripts, most of which had not been previously identified. Nearly one third of the genes showed variations in transcript levels during embryo development, including those encoding enzymes involved in carbon or nitrogen metabolism and hormonemediated signaling pathways. Most AS events were detected during the later stages of embryogenesis, i.e., embryo maturation, dehydration, establishment of dormancy, and at the quiescent state. This induction of AS may be explained by the striking clustering of both splicing-related and ABA-associated factors observed at the late phases of seed development. Seed maturation and desiccation, which involve very specific developmental, hormonal, and biochemical processes, were also examined in arabidopsis (Srinivasan et al., 2016), where RNA-seq profiling was performed on developing and mature seeds. Interestingly, transcription and AS showed opposite trends, with transcription declining during seed maturation, while AS increased. Over a quarter of the loci undergoing AS expressed stage-specific splice variants or showed a marked isoform switch, with a striking $88 \%$ of the detected AS events being absent from the TAIR10 genome annotation. Again, there were no significant changes in total transcript levels of many alternatively spliced genes, 
A

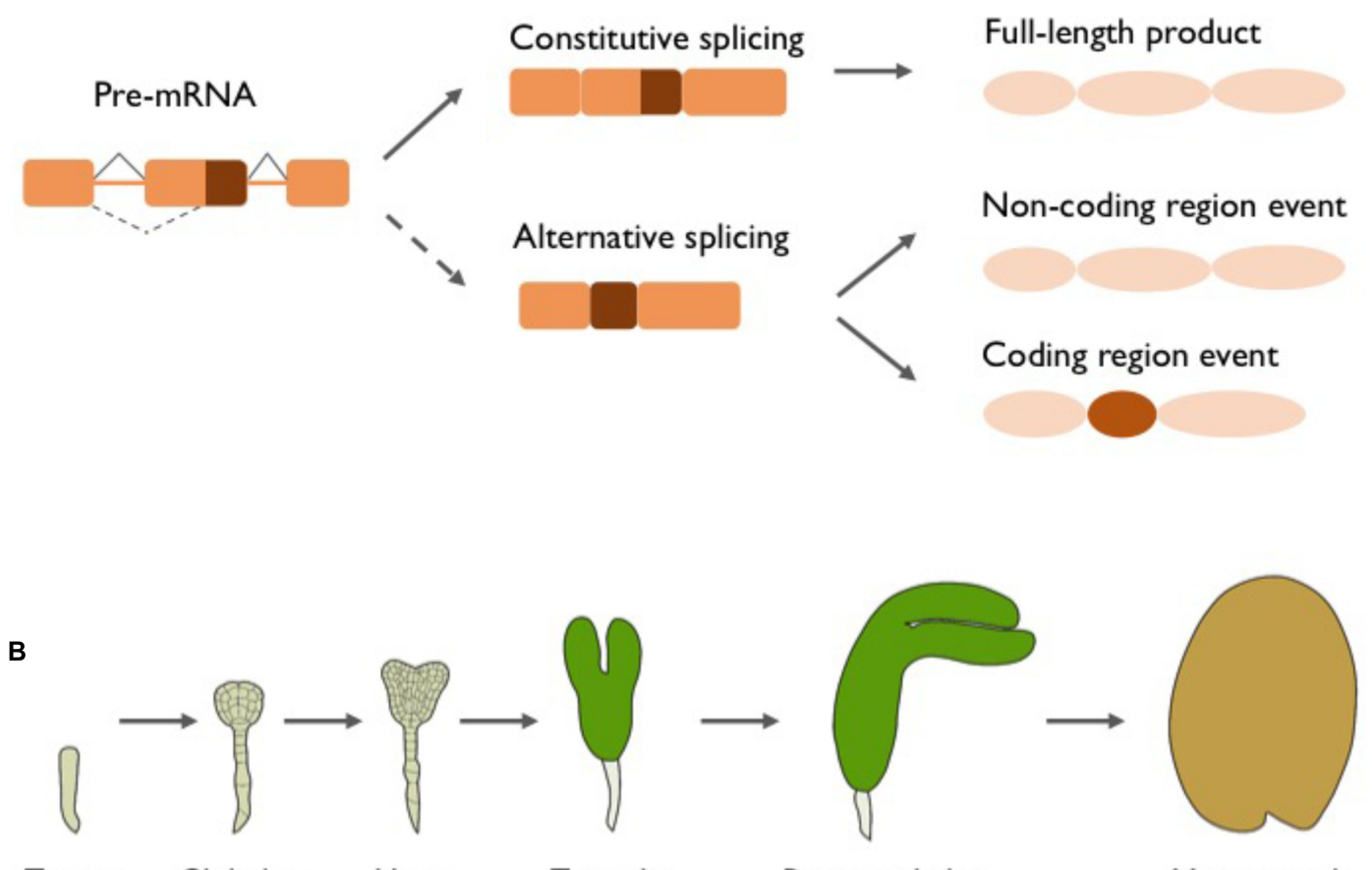

Zygote Globular Heart Torpedo

Bent cotyledon

Mature seed

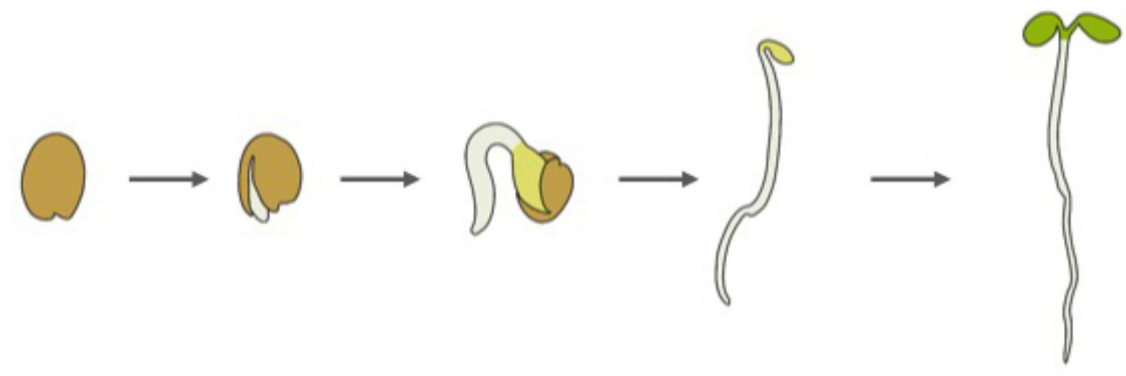

Imbibition Germination Skotomorphogenesis Photomorphogenesis

FIGURE 1 | Alternative splicing and early plant development. (A) Constitutive and alternative splicing. Nascent multi exonic mRNAs need to undergo pre-mRNA splicing. Constitutive splicing removes the non-coding introns, producing a mature mRNA that encodes the full-length protein or transcript with biological functions. The same pre-mRNA molecule can undergo alternative splicing (AS) and produce different transcript variants. AS events occurring in non-coding sequences often impact gene expression, but will result in a protein identical to the full-length isoform. In numerous cases, coding regions are affected by AS, thus originating markedly different mRNAs and potentially distinct proteins that can vary in virtually all functional aspects. (B) Embryogenesis, seed maturation and germination, and early seedling development. After fertilization, the zygote undergoes a rapid succession of highly coordinated cell divisions to form globular stage embryos, which show establishment of the apical-basal axis and a first distinction between outer and inner cells. The embryonic cells further differentiate during the heart stage, when many of the basic cell types (provasculature, endodermis, cortex, and protoderm) and organ primordia (cotyledons, hypocotyl, and primary root) are formed and a bilateral body pattern appears. Expanding cotyledons give the embryo a torpedo shape, and the formation of the shoot and root apical meristems is completed. The next steps involve further cell growth and divisions until the embryo reaches its final shape and size. Seed maturation is completed with the accumulation of reserves and the establishment of desiccation tolerance and seed dormancy. Dry seeds are released from dormancy in response to a combination of environmental cues and internal signals. After water uptake, key biochemical and molecular processes are restored, followed by the rupture of the seed coat and emergence of the radicle, marking the completion of germination. Under darkness, the buried seedling undergoes skotomorphogenesis characterized by a short root, elongated hypocotyl, apical hook and absence of photosynthetic pigments. Upon light exposure, photomorphogenesis is activated leading to inhibition of hypocotyl elongation, opening and expansion of the cotyledons, and initiation of photosynthesis after chloroplast maturation.

pointing to AS as an important regulatory mechanism operating independently from transcription. Most of the genes exhibiting differential splicing were involved in RNA processing, potentially amplifying the AS regulatory effect in preparation for seed germination.
Two recent studies addressed the AS contribution during seed germination. In barley embryos, 14-20\% of multiexon genes expressed multiple mRNA isoforms, some of which displayed clear changes during early germination (Zhang et al., 2016). Surprisingly, the most prominent AS event was alternative $3^{\prime} \mathrm{SS}$ 
selection, and there were no substantial alterations in total transcript levels for most genes. Assessment of the biological functions of the genes undergoing AS during germination indicated involvement in protein synthesis, energy and carbon metabolism as well as RNA transport and splicing. Overall, seed germination appears to require expression of a specific set of genes, with AS playing a widespread role. The regulatory potential of AS during germination is underscored by a subsequent report in arabidopsis (Narsai et al., 2017) confirming the expression of time- and tissue-specific mRNA variants, the occurrence of dynamic changes in isoform abundance, and that splicing regulators are major AS targets during this developmental process.

AS regulation during early plant growth is also relevant in the context of environmental responses. Light, which is perceived by various photoreceptors, strongly impacts the life cycle of plants, regulating among others early developmental steps such as seed germination and the transition to autotrophic growth. Genome-wide effects of light on plant AS were recently analyzed by RNA-seq (Wu et al., 2014; Mancini et al., 2016), including in very young seedlings (Shikata et al., 2014; Hartmann et al., 2016). Shikata et al. (2014) reported that, during the initial response of etiolated seedlings to red light, the number of genes showing phytochrome-mediated differential gene expression or changed AS pattern is comparable, while later transcription becomes the dominant regulatory mechanism. In the phytochrome-dependent AS dataset, splicing-related genes were overrepresented, including SR proteins and the U1 and U2 spliceosomal subunits, while transcription factors comprised the major group of differentially expressed genes. AS seemed to play a significant role in light-induced chloroplast differentiation, as photosynthesis- and plastid-related genes were also enriched in the differential AS sets. When Hartmann et al. (2016) analyzed the response of etiolated arabidopsis seedlings exposed blue, red, or white light treatments, $\sim 20 \%$ of genes were found to be differentially expressed, with $\sim 700$ AS events being detected, most of which mapped to coding sequences. Again, gene ontology analysis revealed overrepresentation of the RNAbinding category, including many splicing factors. A link between light-induced AS and mRNA stability was also uncovered, with $77.2 \%$ of the detected mRNA isoforms more abundant in the dark samples being potential NMD targets. Remarkably, in most of AS events, an isoform switch from a putative instable mRNA variant to a protein-coding alternative occurred upon light exposure. Moreover, mutants lacking the major red or blue light receptors showed impaired AS mainly when subjected to monochromatic red or blue light, indicating that additional signaling pathways influence AS under white light. The authors suggested that metabolic signals, sugars in particular, are implicated in lightmediated AS regulation.

\section{SPLICING FACTORS REGULATING EARLY PLANT DEVELOPMENT}

Compelling evidence from large-scale analyses pointing to an important role for AS during early plant development is being substantiated by accumulating in vivo genetic studies (Table 1). Overexpression or complete abrogation of splicing function often causes embryo lethality, indicating that the corresponding genes are essential for viability and development of a functional plant (Kalyna et al., 2003; Schmitz-Linneweber et al., 2006; Liu et al., 2009; Kim et al., 2010; Fouquet et al., 2011; Swaraz et al., 2011; Perea-Resa et al., 2012; Shikata et al., 2012; Sasaki et al., 2015; Tsugeki et al., 2015). Some studies have established a hormonal basis for the embryo and early seedling development defects caused by altered expression of splicing factors (Kalyna et al., 2003; Casson et al., 2009; Tsugeki et al., 2015), with abnormal spatial distribution of auxin arising from erroneous splicing and expression of auxin biosynthesis, transport, and signaling genes. A link between mRNA splicing and auxin signaling was also uncovered in flowers, where subcellular compartmentation of an auxin biosynthetic gene is regulated by AS (Kriechbaumer et al., 2012).

Seed dormancy and germination are also strongly affected in mRNA processing mutants. These effects were mostly reported to relate to splicing (Dolata et al., 2015) and polyadenylation (Cyrek et al., 2016) of the DOG1 gene, a key seed dormancy regulator and known AS target, and to changes in ABA signaling (Xiong et al., 2001; Sugliani et al., 2010; Jiang et al., 2012). Early seedling development can be affected as a manifestation of wider pleiotropic defects (Liu et al., 2010; Swaraz et al., 2011; Perea-Resa et al., 2012; Shikata et al., 2012; Hsieh et al., 2015; Yap et al., 2015) or in weak alleles of embryo lethal mutants (Kalyna et al., 2003; GutierrezMarcos et al., 2007; Fouquet et al., 2011; Tsugeki et al., 2015). Observed phenotypes include disturbed cotyledons, hypocotyls, vasculature patterning, roots and/or seedling viability and growth. Notably, mRNA splicing in plastids and mitochondria appears to be crucial for seed development and plant growth in both arabidopsis and maize (Schmitz-Linneweber et al., 2006; Gutierrez-Marcos et al., 2007; Liu et al., 2010; Hernando et al., 2015; Hsieh et al., 2015; Yap et al., 2015; Chen et al., 2017).

Genetic and molecular analyses have confirmed a role for splicing factors in photomorphogenesis, particularly in red-light responses. Phytochrome-dependent light signaling influences AS through specific splicing components, with additional splicing factors such as SR proteins being differentially processed in loss-of-function mutants of these effectors under various light conditions (Shikata et al., 2012; Hernando et al., 2015; Xin et al., 2017). Interestingly, Xin et al. (2017) demonstrated red lightdependent direct interaction and colocalization of a splicing factor and phytochrome B.

\section{ALTERNATIVE SPLICING TARGETS AFFECTING EARLY PLANT DEVELOPMENT}

Despite massive transcriptome changes imposed by AS during early plant development, only a handful of alternatively spliced transcripts have had their functional significance analyzed in detail (Table 1). While one group, including the arabidopsis 
TABLE 1 | Splicing factors and targets functioning in early plant development.

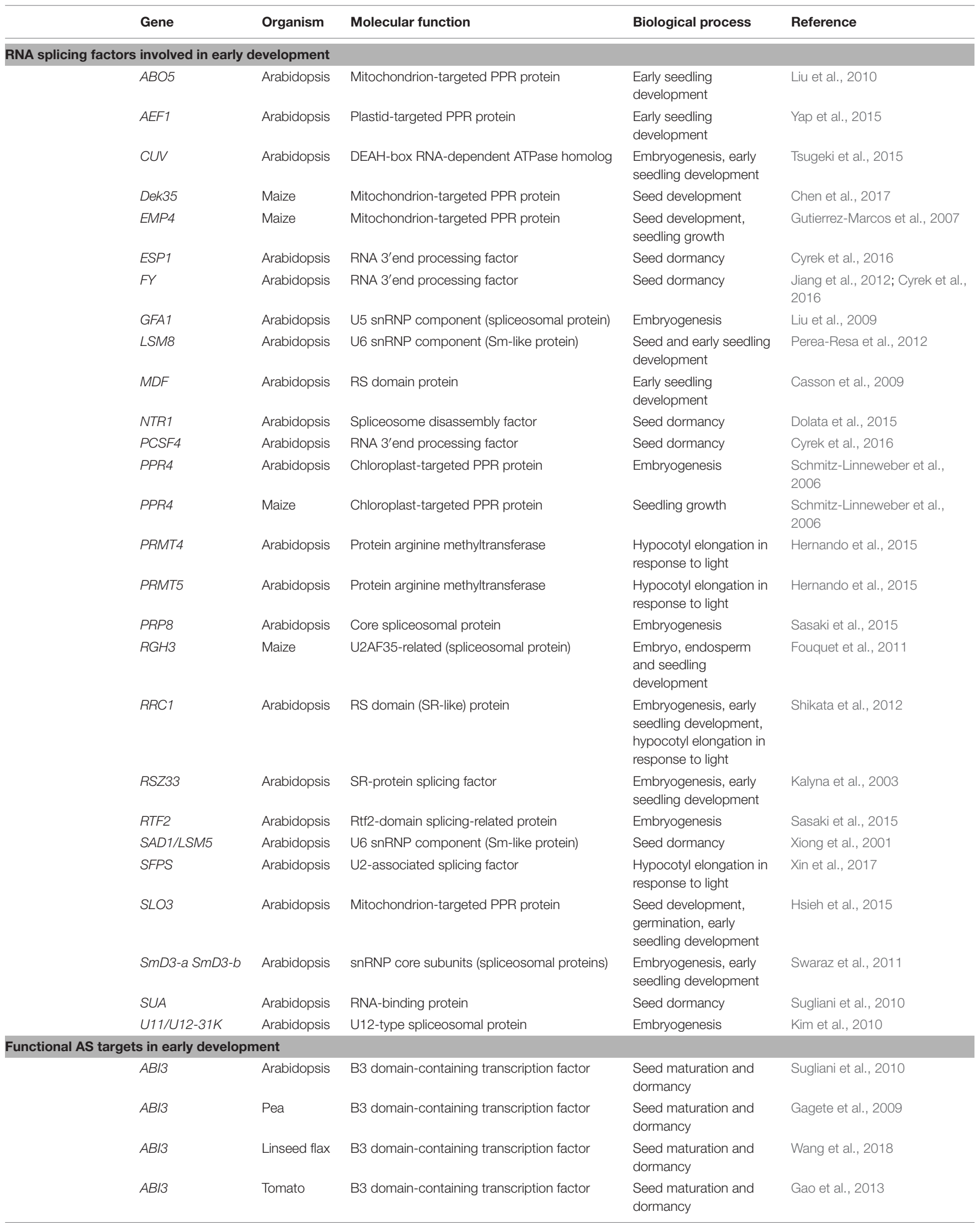


TABLE 1 | Continued

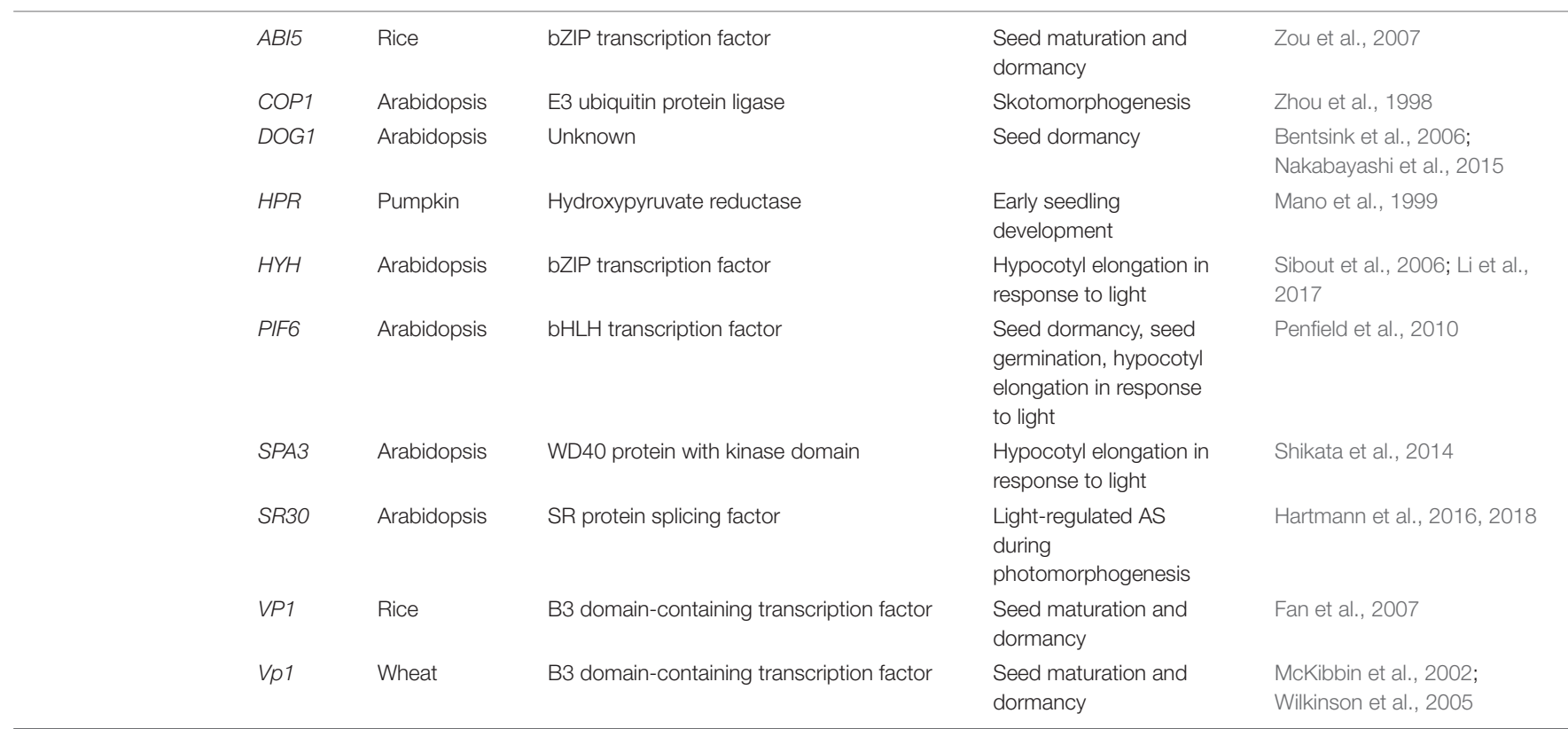

DOG1 gene as well as the $O s A B I 5$ and $A B I 3 / V P 1$ transcription factors, plays roles in seed maturation, dormancy, and ABA responses (McKibbin et al., 2002; Wilkinson et al., 2005; Bentsink et al., 2006; Fan et al., 2007; Zou et al., 2007; Gagete et al., 2009; Sugliani et al., 2010; Gao et al., 2013; Nakabayashi et al., 2015; Wang et al., 2018), another is important for light signaling and includes COP1, HYH, and SPA3 (Zhou et al., 1998; Sibout et al., 2006; Shikata et al., 2014; Li et al., 2017). Moreover, PIF6 regulates both seed dormancy and light responses (Penfield et al., 2010). Despite the few individual events studied, AS is known to act via diverse mechanisms, as illustrated below.

In agreement with results from large-scale studies, the expression of numerous individual mRNA variants was found to be development- or tissue-specific (Zhou et al., 1998; Fan et al., 2007; Gagete et al., 2009; Sugliani et al., 2010; Gao et al., 2013; Wang et al., 2018), with some turning out to be non-functional, either because they did not produce an active protein or no phenotypic consequence was observed as a result of ectopic expression (Wang et al., 2018). In another study, genetic complementation tests indicated that the different splice variants perform functions equivalent to the constitutive form, even when lacking crucial amino acid sequences or domains (Li et al., 2017). Similarly, AS did not fundamentally influence DNA-binding or protein-protein interaction ability of the ABI3 and ABI5 transcription factors from different plant species, though the binding strength appeared to differ among the various isoforms (Zou et al., 2007; Gagete et al., 2009; Gao et al., 2013).

Alternative splice variants can also fulfill similar or distinct functions depending on developmental stage. The constitutive and alternative PIF6 mRNA variants similarly influenced light responses in seedlings, while only the short isoform displayed evident functions during seed germination (Penfield et al., 2010).
In the case of COP1 and SPA3, ectopic overexpression of alternative splice forms phenocopied knock-out mutant phenotypes, indicating that some alternative forms can interfere with the function of the full-length protein (Zhou et al., 1998; Shikata et al., 2014). Strikingly, co-expression and direct protein interactions were found to be necessary for full DOG1 function (Nakabayashi et al., 2015). In genetic complementation assays, independent expression of individual DOG1 isoforms driven by the native promoter did not restore seed dormancy, whereas transgenic lines carrying two or more DOG1 variants showed improved dormancy. Detailed analysis of these results supported the hypothesis that, although single isoforms are active, the presence of multiple isoforms is required for adequate DOG1 function. On the other hand, AS-induced changes in protein sequence may lead not only to diminished biological function but, as demonstrated for an $H Y H$ isoform lacking a protein interaction domain for proteasomal degradation, also to a more stable and hence more active protein isoform (Sibout et al., 2006).

Subcellular targeting provides specialized locations for intracellular processes and can interfere with the regulatory and biochemical potential of proteins. AS of a pumpkin hydroxypyruvate reductase (HPR) acting in photorespiration affected the C-terminal targeting sequence, with one splice form localizing in the peroxisome and another in the cytosol (Mano et al., 1999). The two mRNAs were expressed at similar levels in darkness, while light promoted the production of the shorter, cytosol-localized variant. Most recently, retention of an mRNA variant of the arabidopsis SR30 splicing regulator in the nucleus was shown to influence mRNA stability by preventing the degradation of a potential NMD target in the cytoplasm and its association to the translation machinery (Hartmann et al., 2018). 


\section{CONCLUSION AND PERSPECTIVES}

Recent transcriptome-wide, genetic and molecular studies have demonstrated that regulation of the complex developmental steps from embryogenesis to establishment of a functional plant includes posttranscriptional control via AS. Seed maturation, establishment and maintenance of seed dormancy, and young seedling responses to light stand out as significant AS-regulated processes. The detection of time- and tissue-specific mRNA variants and of notable switches in splicing patterns substantiate crucial roles for AS in other early development processes. Further large-scale analyses in different tissue types using the latest sequencing technologies and single-cell approaches will be key to understand the full extent of AS events occurring during the initial stages of plant development. Improved standardization of data processing and analysis along with more meticulous experimental set-ups should also allow for more reliable comparative studies. Comprehensive publicly available databases, providing a detailed and up-to-date view of AS in plants are still lacking. These will be pivotal in pinpointing promising novel splice forms and assist in functional studies to distinguish biologically relevant AS contributing to proteomic diversity or gene expression regulation from nonfunctional AS events and splicing noise. Importantly, state-ofthe-art methodology such as iCLIP is proving successful in

\section{REFERENCES}

Aghamirzaie, D., Nabiyouni, M., Fang, Y., Klumas, C., Heath, L. S., Grene, R., et al. (2013). Changes in rna splicing in developing soybean (glycine max) embryos. Biology 2, 1311-1337. doi: 10.3390/biology2041311

Bentsink, L., Jowett, J., Hanhart, C. J., and Koornneef, M. (2006). Cloning of DOG1, a quantitative trait locus controlling seed dormancy in Arabidopsis. Proc. Natl. Acad. Sci. U.S.A. 103, 17042-17047. doi: 10.1073/pnas.0607877103

Berger, F., Hamamura, Y., Ingouff, M., and Higashiyama, T. (2008). Double fertilization - caught in the act. Trends Plant Sci. 13, 437-443. doi: 10.1016/j. tplants.2008.05.011

Casson, S. A., Topping, J. F., and Lindsey, K. (2009). MERISTEM-DEFECTIVE, an RS domain protein, is required for the correct meristem patterning and function in Arabidopsis. Plant J. 57, 857-869. doi: 10.1111/j.1365-313X.2008.03738.x

Chamala, S., Feng, G., Chavarro, C., and Barbazuk, W. B. (2015). Genomewide identification of evolutionarily conserved alternative splicing events in flowering plants. Front. Bioeng. Biotechnol. 3:33. doi: 10.3389/fbioe.2015.00033

Chen, X., Feng, F., Qi, W., Xu, L., Yao, D., Wang, Q., et al. (2017). Dek35 Encodes a PPR Protein that Affects cis-Splicing of Mitochondrial nad4 Intron 1 and Seed Development in Maize. Mol. Plant 10, 427-441. doi: 10.1016/j.molp.2016. 08.008

Cyrek, M., Fedak, H., Ciesielski, A., Guo, Y., Sliwa, A., Brzezniak, L., et al. (2016). Seed Dormancy in Arabidopsis Is Controlled by Alternative Polyadenylation of DOG1. Plant Physiol. 170, 947-955. doi: 10.1104/pp.15.01483

Dolata, J., Guo, Y., Kolowerzo, A., Smolinski, D., Brzyzek, G., Jarmolowski, A., et al. (2015). NTR1 is required for transcription elongation checkpoints at alternative exons in Arabidopsis. EMBO J. 34, 544-558. doi: 10.15252/embj.201489478

Drechsel, G., Kahles, A., Kesarwani, A. K., Stauffer, E., Behr, J., Drewe, P., et al. (2013). Nonsense-mediated decay of alternative precursor mRNA splicing variants is a major determinant of the Arabidopsis steady state transcriptome. Plant Cell 25, 3726-3742. doi: 10.1105/tpc.113.115485

Fan, J., Niu, X., Wang, Y., Ren, G., Zhuo, T., Yang, Y., et al. (2007). Short, direct repeats (SDRs)-mediated post-transcriptional processing of a transcription factor gene OsVP1 in rice (Oryza sativa). J. Exp. Bot. 58, 3811-3817. doi: $10.1093 / \mathrm{jxb} / \mathrm{erm} 231$ plant systems and should allow identification of the mRNAs targeted directly by splicing factors to control early plant development.

\section{AUTHOR CONTRIBUTIONS}

All authors listed have made a substantial, direct and intellectual contribution to the work, and approved it for publication.

\section{FUNDING}

DS was supported by a Postdoctoral Fellowship (SFRH/BPD/94796/2013) from Fundação para a Ciência e a Tecnologia (FCT), which also finances research in our lab through Grant PTDC/BIA-PLA/1084/2014. Funding from the GREEN-it research unit (UID/Multi/04551/2013) is also acknowledged.

\section{ACKNOWLEDGMENTS}

Our apologies to the authors whose work was not cited due to space restrictions.

Fouquet, R., Martin, F., Fajardo, D. S., Gault, C. M., Gomez, E., Tseung, C. W., et al. (2011). Maize rough endosperm 3 encodes an RNA splicing factor required for endosperm cell differentiation and has a nonautonomous effect on embryo development. Plant Cell 23, 4280-4297. doi: 10.1105/tpc.111.092163

Gagete, A. P., Riera, M., Franco, L., and Rodrigo, M. I. (2009). Functional analysis of the isoforms of an ABI3-like factor of Pisum sativum generated by alternative splicing. J. Exp. Bot. 60, 1703-1714. doi: 10.1093/jxb/erp038

Gao, Y., Liu, J., Zhang, Z., Sun, X., Zhang, N., Fan, J., et al. (2013). Functional characterization of two alternatively spliced transcripts of tomato ABSCISIC ACID INSENSITIVE3 (ABI3) gene. Plant Mol. Biol. 82, 131-145. doi: 10.1007/ s11103-013-0044-1

Graeber, K., Nakabayashi, K., Miatton, E., Leubner-Metzger, G., and Soppe, W. J. (2012). Molecular mechanisms of seed dormancy. Plant Cell Environ. 35, 1769-1786. doi: 10.1111/j.1365-3040.2012.02542.x

Gutierrez-Marcos, J. F., Dal Pra, M., Giulini, A., Costa, L. M., Gavazzi, G., Cordelier, S., et al. (2007). empty pericarp4 encodes a mitochondrion-targeted pentatricopeptide repeat protein necessary for seed development and plant growth in maize. Plant Cell 19, 196-210. doi: 10.1105/tpc.105.039594

Hartmann, L., Drewe-Boss, P., Wiessner, T., Wagner, G., Geue, S., Lee, H. C., et al. (2016). Alternative splicing substantially diversifies the transcriptome during early photomorphogenesis and correlates with the energy availability in Arabidopsis. Plant Cell 28, 2715-2734. doi: 10.1105/tpc.16.0 0508

Hartmann, L., Wießner, T., and Wachter, A. (2018). Subcellular compartmentation of alternatively-spliced transcripts defines SERINE/ARGININE-RICH PROTEIN30 expression. Plant Physiol. 176, 2886-2903. doi: 10.1104/pp. 17.01260

Hernando, C. E., Sanchez, S. E., Mancini, E., and Yanovsky, M. J. (2015). Genome wide comparative analysis of the effects of PRMT5 and PRMT4/CARM1 arginine methyltransferases on the Arabidopsis thaliana transcriptome. BMC Genomics 16:192. doi: 10.1186/s12864-015-1399-2

Hsieh, W. Y., Liao, J. C., Chang, C. Y., Harrison, T., Boucher, C., and Hsieh, M. H. (2015). The SLOW GROWTH3 pentatricopeptide repeat protein is required for the splicing of Mitochondrial NADH dehydrogenase subunit7 intron 2 in Arabidopsis. Plant Physiol. 168, 490-501. doi: 10.1104/pp.15.00354 
Iniguez, L. P., Ramirez, M., Barbazuk, W. B., and Hernandez, G. (2017). Identification and analysis of alternative splicing events in Phaseolus vulgaris and glycine max. BMC Genomics 18:650. doi: 10.1186/s12864-017-4054-2

Jiang, S., Kumar, S., Eu, Y. J., Jami, S. K., Stasolla, C., and Hill, R. D. (2012). The Arabidopsis mutant, fy-1, has an ABA-insensitive germination phenotype. J. Exp. Bot. 63, 2693-2703. doi: 10.1093/jxb/err452

Kalyna, M., Lopato, S., and Barta, A. (2003). Ectopic expression of atRSZ33 reveals its function in splicing and causes pleiotropic changes in development. Mol. Biol. Cell 14, 3565-3577. doi: 10.1091/mbc.E03-02-0109

Kalyna, M., Simpson, C. G., Syed, N. H., Lewandowska, D., Marquez, Y., Kusenda, B., et al. (2012). Alternative splicing and nonsense-mediated decay modulate expression of important regulatory genes in Arabidopsis. Nucleic Acids Res. 40, 2454-2469. doi: 10.1093/nar/gkr932

Kim, W. Y., Jung, H. J., Kwak, K. J., Kim, M. K., Oh, S. H., Han, Y. S., et al. (2010). The Arabidopsis U12-type spliceosomal protein U11/U12-31K is involved in U12 intron splicing via RNA chaperone activity and affects plant development. Plant Cell 22, 3951-3962. doi: 10.1105/tpc.110.079103

Klepikova, A. V., Kasianov, A. S., Gerasimov, E. S., Logacheva, M. D., and Penin, A. A. (2016). A high resolution map of the Arabidopsis thaliana developmental transcriptome based on RNA-seq profiling. Plant J. 88, 1058-1070. doi: 10.1111/ tpj. 13312

Kriechbaumer, V., Wang, P., Hawes, C., and Abell, B. M. (2012). Alternative splicing of the auxin biosynthesis gene YUCCA4 determines its subcellular compartmentation. Plant J. 70, 292-302. doi: 10.1111/j.1365-313X.2011.04866.

Kucera, B., Cohn, M. A., and Leubner-Metzger, G. (2005). Plant hormone interactions during seed dormancy release and germination. Seed Sci. Res. 15, 281-307. doi: 10.1079/ssr2005218

Laloum, T., Martin, G., and Duque, P. (2017). Alternative Splicing Control of Abiotic Stress Responses. Trends Plant Sci 23, 140-150. doi: 10.1016/j.tplants. 2017.09.019

Li, C., Zheng, L., Zhang, J., Lv, Y., Liu, J., Wang, X., et al. (2017). Characterization and functional analysis of four HYH splicing variants in Arabidopsis hypocotyl elongation. Gene 619, 44-49. doi: 10.1016/j.gene.2017.04.001

Liu, M., Yuan, L., Liu, N. Y., Shi, D. Q., Liu, J., and Yang, W. C. (2009). GAMETOPHYTIC FACTOR 1, involved in pre-mRNA splicing, is essential for megagametogenesis and embryogenesis in Arabidopsis. J. Integr. Plant Biol. 51, 261-271. doi: 10.1111/j.1744-7909.2008.00783.x

Liu, Y., He, J., Chen, Z., Ren, X., Hong, X., and Gong, Z. (2010). ABA overlysensitive 5 (ABO5), encoding a pentatricopeptide repeat protein required for cis-splicing of mitochondrial nad2 intron 3, is involved in the abscisic acid response in Arabidopsis. Plant J. 63, 749-765. doi: 10.1111/j.1365-313X.2010. 04280.x

Lu, T., Lu, G., Fan, D., Zhu, C., Li, W., Zhao, Q., et al. (2010). Function annotation of the rice transcriptome at single-nucleotide resolution by RNA-seq. Genome Res. 20, 1238-1249. doi: 10.1101/gr.106120.110

Lu, X., Chen, D., Shu, D., Zhang, Z., Wang, W., Klukas, C., et al. (2013). The differential transcription network between embryo and endosperm in the early developing maize seed. Plant Physiol. 162, 440-455. doi: 10.1104/pp.113.214874

Mancini, E., Sanchez, S. E., Romanowski, A., Schlaen, R. G., Sanchez-Lamas, M., Cerdan, P. D., et al. (2016). Acute effects of light on alternative splicing in light-grown plants. Photochem. Photobiol. 92, 126-133. doi: 10.1111/php.12550

Mano, S., Hayashi, M., and Nishimura, M. (1999). Light regulates alternative splicing of hydroxypyruvate reductase in pumpkin. Plant J. 17, 309-320. doi: 10.1046/j.1365-313X.1999.00378.x

Marquez, Y., Brown, J. W., Simpson, C., Barta, A., and Kalyna, M. (2012). Transcriptome survey reveals increased complexity of the alternative splicing landscape in Arabidopsis. Genome Res. 22, 1184-1195. doi: 10.1101/gr.134106. 111

McKibbin, R. S., Wilkinson, M. D., Bailey, P. C., Flintham, J. E., Andrew, L. M., Lazzeri, P. A., et al. (2002). Transcripts of Vp-1 homeologues are misspliced in modern wheat and ancestral species. Proc. Natl. Acad. Sci. U.S.A. 99, 10203-10208. doi: 10.1073/pnas.152318599

Meyer, K., Koester, T., and Staiger, D. (2015). Pre-mRNA splicing in plants: in Vivo functions of RNA-binding proteins implicated in the splicing process. Biomolecules 5, 1717-1740. doi: 10.3390/biom5031717

Nakabayashi, K., Bartsch, M., Ding, J., and Soppe, W. J. (2015). Seed dormancy in Arabidopsis requires self-binding ability of $\operatorname{dog} 1$ protein and the presence of multiple isoforms generated by alternative splicing. PLoS Genet. 11:e1005737. doi: 10.1371/journal.pgen.1005737

Narsai, R., Gouil, Q., Secco, D., Srivastava, A., Karpievitch, Y. V., Liew, L. C., et al. (2017). Extensive transcriptomic and epigenomic remodelling occurs during Arabidopsis thaliana germination. Genome Biol. 18:172. doi: 10.1186/s13059017-1302-3

Nee, G., Xiang, Y., and Soppe, W. J. (2017). The release of dormancy, a wake-up call for seeds to germinate. Curr. Opin. Plant Biol. 35, 8-14. doi: 10.1016/j.pbi. 2016.09.002

Palovaara, J., De Zeeuw, T., and Weijers, D. (2016). Tissue and organ initiation in the plant embryo: a first time for everything. Annu. Rev. Cell Dev. Biol. 32, 47-75. doi: 10.1146/annurev-cellbio-111315-124929

Penfield, S., Josse, E. M., and Halliday, K. J. (2010). A role for an alternative splice variant of PIF6 in the control of Arabidopsis primary seed dormancy. Plant Mol. Biol. 73, 89-95. doi: 10.1007/s11103-009-9571-1

Perea-Resa, C., Hernandez-Verdeja, T., Lopez-Cobollo, R., Del Mar Castellano, M., and Salinas, J. (2012). LSM proteins provide accurate splicing and decay of selected transcripts to ensure normal Arabidopsis development. Plant Cell 24, 4930-4947. doi: 10.1105/tpc.112.103697

Proudfoot, N. J. (2011). Ending the message: poly(A) signals then and now. Genes Dev. 25, 1770-1782. doi: 10.1101/gad.17268411

Qu, J., Ma, C., Feng, J., Xu, S., Wang, L., Li, F., et al. (2016). Transcriptome dynamics during maize endosperm development. PLoS One 11:e0163814. doi: 10.1371/journal.pone.0163814

Raghavan, V. (2003). Some reflections on double fertilization, from its discovery to the present. New Phytol. 159, 565-583. doi: 10.1046/j.1469-8137.2003. 00846.x

Ramanathan, A., Robb, G. B., and Chan, S. H. (2016). mRNA capping: biological functions and applications. Nucleic Acids Res. 44, 7511-7526. doi: 10.1093/nar/ gkw551

Reddy, A. S., Marquez, Y., Kalyna, M., and Barta, A. (2013). Complexity of the alternative splicing landscape in plants. Plant Cell 25, 3657-3683. doi: 10.1105/ tpc.113.117523

Sasaki, T., Kanno, T., Liang, S. C., Chen, P. Y., Liao, W. W., Lin, W. D., et al. (2015). An Rtf2 domain-containing protein influences pre-mRNA splicing and is essential for embryonic development in Arabidopsis thaliana. Genetics 200, 523-535. doi: 10.1534/genetics.115.176438

Schmitz-Linneweber, C., Williams-Carrier, R. E., Williams-Voelker, P. M., Kroeger, T. S., Vichas, A., and Barkan, A. (2006). A pentatricopeptide repeat protein facilitates the trans-splicing of the maize chloroplast rps12 pre-mRNA. Plant Cell 18, 2650-2663. doi: 10.1105/tpc.106.046110

Shang, X., Cao, Y., and Ma, L. (2017). Alternative splicing in plant genes: a means of regulating the environmental fitness of plants. Int. J. Mol. Sci. 18:E432. doi: 10.3390/ijms18020432

Shen, Y., Zhou, Z., Wang, Z., Li, W., Fang, C., Wu, M., et al. (2014). Global dissection of alternative splicing in paleopolyploid soybean. Plant Cell 26, 996-1008. doi: 10.1105/tpc.114.122739

Shi, Y., and Manley, J. L. (2015). The end of the message: multiple protein-RNA interactions define the mRNA polyadenylation site. Genes Dev. 29, 889-897. doi: $10.1101 / \operatorname{gad} .261974 .115$

Shikata, H., Hanada, K., Ushijima, T., Nakashima, M., Suzuki, Y., and Matsushita, T. (2014). Phytochrome controls alternative splicing to mediate light responses in Arabidopsis. Proc. Natl. Acad. Sci. U.S.A. 111, 18781-18786. doi: $10.1073 /$ pnas. 1407147112

Shikata, H., Shibata, M., Ushijima, T., Nakashima, M., Kong, S. G., Matsuoka, K., et al. (2012). The RS domain of Arabidopsis splicing factor RRC1 is required for phytochrome B signal transduction. Plant J. 70, 727-738. doi: 10.1111/j.1365313X.2012.04937.x

Sibout, R., Sukumar, P., Hettiarachchi, C., Holm, M., Muday, G. K., and Hardtke, C. S. (2006). Opposite root growth phenotypes of hy5 versus hy5 hyh mutants correlate with increased constitutive auxin signaling. PLoS Genet. 2:e202. doi: 10.1371/journal.pgen.0020202

Srinivasan, A., Jimenez-Gomez, J. M., Fornara, F., Soppe, W. J., and Brambilla, V. (2016). Alternative splicing enhances transcriptome complexity in desiccating seeds. J. Integr. Plant Biol. 58, 947-958. doi: 10.1111/jipb.12482

Staiger, D., and Brown, J. W. (2013). Alternative splicing at the intersection of biological timing, development, and stress responses. Plant Cell 25, 3640-3656. doi: $10.1105 /$ tpc. 113.113803 
Sugliani, M., Brambilla, V., Clerkx, E. J., Koornneef, M., and Soppe, W. J. (2010). The conserved splicing factor SUA controls alternative splicing of the developmental regulator ABI3 in Arabidopsis. Plant Cell 22, 1936-1946. doi: $10.1105 /$ tpc. 110.074674

Sun, Y., and Xiao, H. (2015). Identification of alternative splicing events by RNA sequencing in early growth tomato fruits. BMC Genomics 16:948. doi: 10.1186/ s12864-015-2128-6

Swaraz, A. M., Park, Y. D., and Hur, Y. (2011). Knock-out mutations of Arabidopsis SmD3-b induce pleotropic phenotypes through altered transcript splicing. Plant Sci. 180, 661-671. doi: 10.1016/j.plantsci.2011. 01.011

Thatcher, S. R., Danilevskaya, O. N., Meng, X., Beatty, M., Zastrow-Hayes, G., Harris, C., et al. (2016). Genome-wide analysis of alternative splicing during development and drought stress in maize. Plant Physiol. 170, 586-599. doi: 10.1104/pp.15.01267

Thatcher, S. R., Zhou, W., Leonard, A., Wang, B. B., Beatty, M., ZastrowHayes, G., et al. (2014). Genome-wide analysis of alternative splicing in Zea mays: landscape and genetic regulation. Plant Cell 26, 3472-3487. doi: 10.1105/ tpc. 114.130773

Tsugeki, R., Tanaka-Sato, N., Maruyama, N., Terada, S., Kojima, M., Sakakibara, H., et al. (2015). CLUMSY VEIN, the Arabidopsis DEAH-box Prp16 ortholog, is required for auxin-mediated development. Plant J. 81, 183-197. doi: 10.1111/ tpj. 12721

Vaneechoutte, D., Estrada, A. R., Lin, Y. C., Loraine, A. E., and Vandepoele, K. (2017). Genome-wide characterization of differential transcript usage in Arabidopsis thaliana. Plant J. 92, 1218-1231. doi: 10.1111/tpj. 13746

Wang, Y., Zhang, T., Song, X., Zhang, J., Dang, Z., Pei, X., et al. (2018). Identification and functional analysis of two alternatively spliced transcripts of ABSCISIC ACID INSENSITIVE3 (ABI3) in linseed flax (Linum usitatissimum L.). PLoS One 13:e0191910. doi: 10.1371/journal.pone. 0191910

Wilkinson, M., Lenton, J., and Holdsworth, M. (2005). Transcripts of Vp-1 homoeologues are alternatively spliced within the Triticeae tribe. Euphytica 143 , 243-246. doi: 10.1007/s10681-005-7856-2

Wu, H. P., Su, Y. S., Chen, H. C., Chen, Y. R., Wu, C. C., Lin, W. D., et al. (2014). Genome-wide analysis of light-regulated alternative splicing mediated by photoreceptors in Physcomitrella patens. Genome Biol. 15:R10. doi: 10.1186/ gb-2014-15-1-r10

Wu, S. H. (2014). Gene expression regulation in photomorphogenesis from the perspective of the central dogma. Annu. Rev. Plant Biol. 65, 311-333. doi: 10.1146/annurev-arplant-050213-040337
Xin, R., Zhu, L., Salome, P. A., Mancini, E., Marshall, C. M., Harmon, F. G., et al. (2017). SPF45-related splicing factor for phytochrome signaling promotes photomorphogenesis by regulating pre-mRNA splicing in Arabidopsis. Proc. Natl. Acad. Sci. U.S.A. 114, E7018-E7027. doi: 10.1073/pnas.1706379114

Xiong, L., Gong, Z., Rock, C. D., Subramanian, S., Guo, Y., Xu, W., et al. (2001). Modulation of abscisic acid signal transduction and biosynthesis by an $\mathrm{Sm}-$ like protein in Arabidopsis. Dev. Cell 1, 771-781. doi: 10.1016/S1534-5807(01) 00087-9

Yang, S., Tang, F., and Zhu, H. (2014). Alternative splicing in plant immunity. Int. J. Mol. Sci. 15, 10424-10445. doi: 10.3390/ijms150610424

Yap, A., Kindgren, P., Colas Des Francs-Small, C., Kazama, T., Tanz, S. K., Toriyama, K., et al. (2015). AEF1/MPR25 is implicated in RNA editing of plastid atpF and mitochondrial nad5, and also promotes atpF splicing in Arabidopsis and rice. Plant J. 81, 661-669. doi: 10.1111/tpj.12756

Zhang, G., Guo, G., Hu, X., Zhang, Y., Li, Q., Li, R., et al. (2010). Deep RNA sequencing at single base-pair resolution reveals high complexity of the rice transcriptome. Genome Res. 20, 646-654. doi: 10.1101/gr.100677.109

Zhang, Q., Zhang, X., Wang, S., Tan, C., Zhou, G., and Li, C. (2016). Involvement of alternative splicing in barley seed germination. PLoS One 11:e0152824. doi: 10.1371/journal.pone.0152824

Zhang, R., Calixto, C. P. G., Marquez, Y., Venhuizen, P., Tzioutziou, N. A., Guo, W., et al. (2017). A high quality Arabidopsis transcriptome for accurate transcriptlevel analysis of alternative splicing. Nucleic Acids Res. 45, 5061-5073. doi: $10.1093 / \mathrm{nar} / \mathrm{gkx} 267$

Zhou, D. X., Kim, Y. J., Li, Y. F., Carol, P., and Mache, R. (1998). COP1b, an isoform of COP1 generated by alternative splicing, has a negative effect on COP1 function in regulating light-dependent seedling development in Arabidopsis. Mol. Gen. Genet. 257, 387-391. doi: 10.1007/s004380050662

Zou, M., Guan, Y., Ren, H., Zhang, F., and Chen, F. (2007). Characterization of alternative splicing products of bZIP transcription factors OsABI5. Biochem. Biophys. Res. Commun. 360, 307-313. doi: 10.1016/j.bbrc.2007.05.226

Conflict of Interest Statement: The authors declare that the research was conducted in the absence of any commercial or financial relationships that could be construed as a potential conflict of interest.

Copyright (c) 2018 Szakonyi and Duque. This is an open-access article distributed under the terms of the Creative Commons Attribution License (CC BY). The use, distribution or reproduction in other forums is permitted, provided the original author(s) and the copyright owner(s) are credited and that the original publication in this journal is cited, in accordance with accepted academic practice. No use, distribution or reproduction is permitted which does not comply with these terms. 